\title{
LAMP ROBOT WITH MEMORY METAL DRIVES
}

\author{
P.A. BESSELINK, A.W.J. van ROERMUND*, P. KURVER ${ }^{* *}$ and N.W. BUYS*** \\ Memory Metal Holland, Postbox 6066, 7503 GB Enschede, The Netherlands \\ *IPS, Willem Pyperstraat 31, 1077 XL Amsterdam, The Netherlands \\ **BMA, Zandweg 196, 3454 HE De Meern, The Netherlands \\ ***Rijksstraatweg 25, 3454 JA De Meem, The Netherlands
}

\begin{abstract}
.
A lamp robot that obeys verbal orders. The principle of the lamp is basically a combination of two new technologies: speech recognition and memory metal.

The lamp can move in six directions, and the movements can be generated by extremely small memory metal elements with a total mass of about 3 grams. The temperature of the memory elements and thus also their shape can be remote controlled by a system which exists of a Personal Computer with a speech recognition card and a microphone, an interface with $\mathrm{D} / \mathrm{A}$ and $\mathrm{A} / \mathrm{D}$-converter and amplifier and the lamp robot itself.

Heating of the memory metal is achieved by direct resistance heating pulses from the interface. The position of each drive system is recorded continiously by miniature precision potentiometers and any desired intermediate position can be reached and kept by spoken commands. A fast transformation of the TiNi-elements and so short response times is achieved by the use of very thin flat rolled wire of $0.2 * 1.7 \mathrm{~mm}$, produced by Memory Metal Holland in Enschede.

This flat wire combines high recovery forces with much higher cooling rates than round wire.

The lamp robot has been developed for and installed in "The House of the Future" in Rosmalen. The Netherlands, which gives a permanent exhibition of possible applications of new technologies in houses.

A possible application is for use by disabled persons.
\end{abstract}

Introduction.

There were two reasons to develop a Lamp-robot.

The first reason was to show the possibilities of memory metal.

Therefore a permanent demonstration in The House of the Future, where more than half a million visitors come yearly seemed to be a good promotion.

Another significance of the robot lamp is that it demonstrates that for many categories of handicapped technological solutions for the operation of many objects are being offered.

The same principle can be used to open curtains, to switch on the oven, 
to open the door, etc.

The National Rehabilitation Fund and the Organisation for Fund Raising Activities in The Netherlands also recognized this major significance for the handicapped.

These two organisations have paid the development costs of the robot lamp.

\section{Realisation.}

This project has been realised by the following persons.

The idea, integration of speech recognition and memory metal (for which patent was applied) is from Ing. N.W. Buys, De Meern. The lamp has been designed by Ion van Roermund and Ir. Peter Besselink. Ton van Roermund (IPS, Amsterdam) is an industrial designer. He designed the lamp model and the mechanism of the swing arm. Peter Besselink ( Memory Metal Holland. Enschede ) is specialist in the development and manufacturing of memory metal and applications.

He designed the rotation mechanisms and built the lamprobot.

The software and interface which made the control of the robot by means of speech recognition possible have been developed by Ir. P.Kurver (BMA in De Meern, and his team of coworkers.

The complete system (see fig.1) consists of a PC, a speech recognition card, an interface and the lamp itself.

speech recognition means that through an inserted card the computer is capable of understanding spoken commands.

This speech recognition is speaker dependant, i.e. that the PC only listens to persons whose voices he has come to know through a short training programme. A user has to complete such a training programme ( which takes two minutes) only once.

Functions of the lamp.

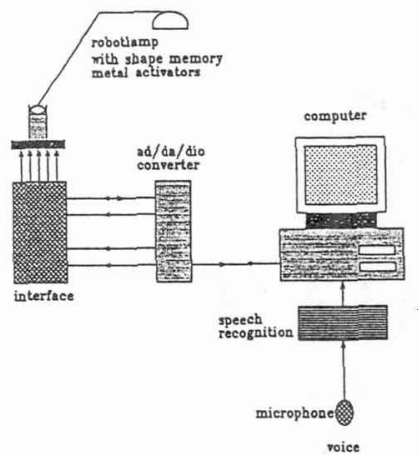

Fig.I scheme of the system.

The lamp can move in six directions. The movements are generated by extremely small memory metal elements with a total mass of about 3 grams. These memory metal elements are made from a Titanium-NickelCopper alloy, which was supplied by GST in Essen, Germany as $8 \mathrm{~mm}$ rod. The wire drawing, strip rolling and production of springs were done by MMH.

The As-temperature of the memory metal elements is 58 degrees celsius. Using memory metal offers the possibility to make the very simple drive systems which are used in this lamp.

In this construction the memory metal is used to imitate muscle functions, where the necessary temperature rise is obtained by conducting electric current directly through the memory wire.

The temperature of the memory elements and thus also their shape can be remote controlled, and the lamp robot can listen to the spoken commands "on", "out", "dim", "clear", "Ieft", "right", "up", "down", "forward", "backward" "stop" and "object".

With this last command several functions of the lamp are combined and it moves to a predefined position where it lights a painting on the wall, for instance.

All mechanisms can be fixed in any desired intermediate position with the command "stop". 

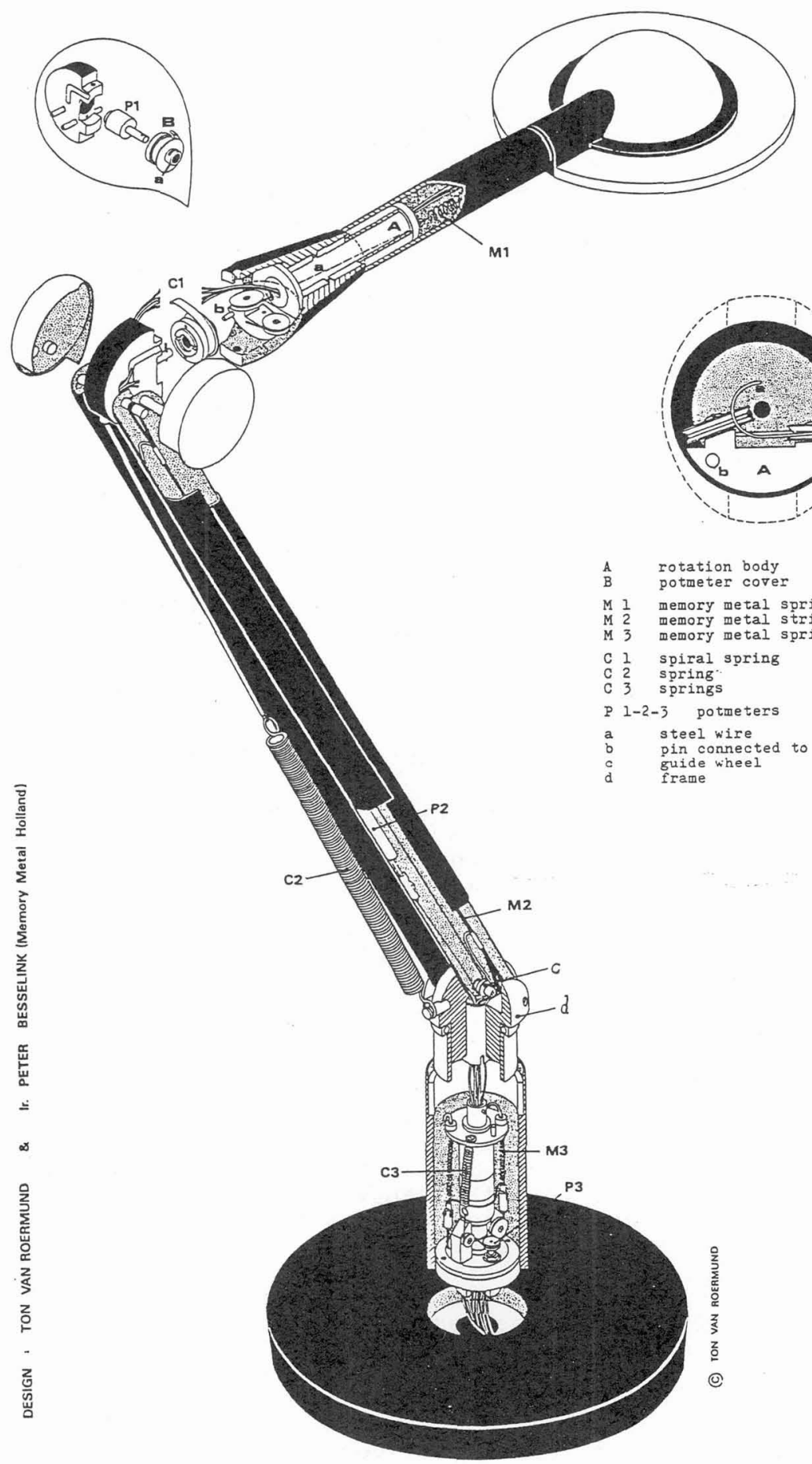

Fig. 2 The lamp robot 
The positien at that moment is monitored by 3 miniature precision potentiometers for respectively the upper arm with the light, the swing arm and the foot rotation.

These positions are maintained because the computer continiously sends digital information to the interface which converts this information into electrical pulses that are amplified to the correct heating energy for the memory elements. By the interface the konstant control voltage for the potentiometers is also supplied.

The temperature of the memory elements can be controlled very accurately and any deviation of the position is corrigated immedetially by the computer.

\section{Construction of the $1 \mathrm{amp}$.}

The drawing of the lamp mechanism makes clear how it works (see fig.2) There are memory metal elements M1, M2 and M3, counteracting steel springs $\mathrm{C} 1, \mathrm{C} 2$ and $\mathrm{C} 3$ and precision pótentiometers $\mathrm{P} 1, \mathrm{P} 2$ and $\mathrm{P} 3$ for upper arm, swing arm and foot rotation respectively.

Upper arm.

The rotation of the upper arm is caused by the two counteractingsprings : memory spring MI and spiral spring $C 1$. The memory spring is connected to a steel wire (a). that runs through the tube of the rotation body (A) and over two guide weels.

The end is attached to the cylindrical part of the potmeter cover (B), which is made clear in the detail at right.

When the memory spring is heated it contracts and the upper arm turns counter-clockwise (see detail above left). The reverse movement is caused by spring $C 1$, that is wrapped around the middle section of the potmeter cover ( $B)$ and mounted on $p$ in $b$ on the rotation body.

Potmeter $P 1$ is connected to the rotation body $A$, so the rotation is directly monitored.

When the electric current is interrupted, the memory spring cools down, becomes weak and thus looses its tractive force. The tractive force of the spiral spring becomes greater than the tractive force of the memory spring and the upper arm turns back clockwise.

Foot rotation.

The same principle goes for the footrotation-mechanism, which contains two memory metal springs (M3) and two counteracting steel springs (C3). All springs are connected to steel wires, that run over small guide weels to the hollow axle of the rotation mechanism: these wires are connected tangentially to this axle and they are attached in such a way that the foot rotates counterclockwise by heating and clockwise by cooling.

Potmeter P3 is driven by the axle by means of a rubber belt.

This mechanism has been designed to transform a iniar movement of contracting memory metal elements into a rotation of the tube, where it is connected with. Parallel with the length axis of the memory elements lies the rotation axis, which makes it possible to construct a long, thin. stand-alone rotating actuator that can be put into a cylindrical place.

When the same movement had to be made with a memory metal torsion element. it would take a much heavier element to reach the same torque and then the drive current and the cooling time would be much longer.

Swing-arm.

The swing-arm is a special double parallelogram construction of four Uprofiles fitting into each other two by two, which makes a perfect horizontal movement of the upper arm possible during rotation of the swing-arm. 
It works with two counteractive tractive systems, that pull the arm to a certain angle: The two memory metal strips M2 pull the arm forward and the steel spring $\mathrm{C} 2$ pulls the arm backward during cooling.

It only takes a contraction of $1 \%$ of the length of the memory metal strips to get a rotation of 25 degrees of the swing-arm.

Using thin. flat rolled strip brings the advantage of the combination of high recovery forces and short temperature cycling times, compared to normal round wire or springs.

A liniar potentiometer P2 is connected to a steel wire that is partly wrapped around a small wheel $c$ below and connected to the frame at $d$.

When the swing-arm moves forward, the steel wire rolls partly over the wheel $c$ and moves the potentiometer rod.

This rod is pulled back by a miniature pseudoelastic spring which is placed above $P 2$ in the u-profile (not visible).

The reach angle of this lamp robot is about 180 degrees for the upper $\mathrm{arm}, 70$ degrees for the swing-arm and 100 degrees for the foot.

\section{Results.}

In figure 3 the position of the swing-arm mechanism is given (in degrees) as a function of time (upper part). The lower part gives the drive signals as voltage levels marked with *. These voltage levels are compared with the desired potentiometer output of the swing-arm position control.

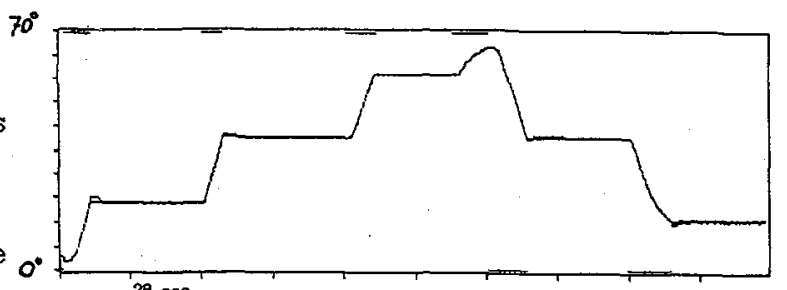

vel is given (during a movement forward) until the measured position equals the desired value and than a minimum signal is given at time $t 2$ to compensate the overshoot. Then, after $t 3$ the hold

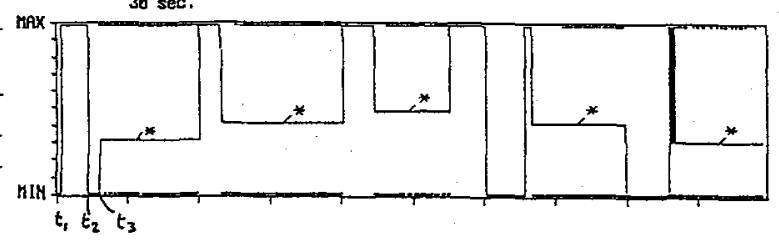
current is given as pulses with a frequency of $18 \mathrm{~Hz}$.

It is an on/off control system which works very simple and accurate.

\section{Conclusion.}

The lamp-robot, based on three different drive systems has been realised to be used as a demonstration object to show some important possibilities by the use of memory metal, in combination with speech recognition.

The performance of the lamp makes that this goal is realised very well. 